\title{
P-112
}

\section{Clinical Study of Axillary Hair Retardation Product Containing Thai Herb "Wan-Ma-Ha-Mek"}

\author{
Jukkarin Srivilai $^{1,{ }^{*}}$, Neti Waranuch ${ }^{2}$, Nantaka Khorana ${ }^{1}$ and Kornkanok Ingkaninan ${ }^{1}$ \\ ${ }^{1}$ Bioscreening Unit, Department of Pharmaceutical Chemistry and Pharmacognosy, Faculty of Pharmaceutical Sciences \\ and Center of Excellence for Innovation in Chemistry, Naresuan University, Phitsanulok 65000, Thailand; ${ }^{2}$ Cosmetic \\ and Natural Product Research Center, Faculty of Pharmaceutical Sciences, Naresuan University, Phitsanulok , 65000, \\ Thailand; E-mail: JSrivilai@gmail.com
}

The development of hair growth is related to androgen activity which play important role to develop secondary sex characteristic. High androgenic activity leads to the growth of body hair in males, therefore anti-androgenic compounds might be good candidates for axillary hair retardation products. Previously, we reported the anti-androgenic activity of a Thai medicinal plant, Wan-Ma-Ha-Mek. In this study, we aimed to develop an axillary hair retardation product containing Wan-MaHa-Mek. To evaluate the efficacy of the product, a double-blinded randomized controlled trial was performed on 30 women (aged 20-60 years). The product or placebo was applied under armpit twice a day for 3 months. Hair growth rate, hair density, mean and culmulative hair lenth were evaluated.

Keywords: Axillary hair, Wan-Ma-Ha-Mek, clinical trial, axillary hair retardation product, anti-androgen. 